Edinilsa Ramos de Souza ${ }^{1}$

Romeu Gomes ${ }^{1}$

Juliana Guimarães e Silva ${ }^{1}$

Bruna Soares Chaves Correia ${ }^{1}$

Marta Maria Alves da Silva ${ }^{2}$

${ }^{1}$ Centro Latino Americano de Estudos sobre Violência e Saúde Jorge Carelli, Escola Nacional de Saúde Pública Sergio Arouca, Fundação Oswaldo Cruz. Av. Brasil 4036/700, Manguinhos. 21.040-361 Rio de Janeiro RJ. edinilsaramos@gmail.com 2 Área Técnica de Vigilância e Prevenção de Violências e Acidentes, Coordenação Geral de Doenças e Agravos não Transmissíveis,

Departamento de Análise de Situação em Saúde,

Secretaria de Vigilância em Saúde, Ministério da Saúde.

\title{
Morbimortalidade de homens jovens brasileiros por agressão: expressão dos diferenciais de gênero
}

\author{
Morbidity and mortality of young Brazilian men \\ due to aggression: expression of gender differentials
}

Abstract Mortality, hospitalization and emergency attendance visits for assault in Brazil, from 1996 to 2007 were analyzed. The data sources are the Mortality Information System/SIM, the Hospital Information System/SIH and the Surveillance System of Violence and Injuries/VIVA of the Ministry of Health. It was focused on males in the 15 to 29 year-old age group, and other variables related to the victim, the aggressor and the event. The male/ woman distribution was 11.6 times higher for mortality, 4.5 times for hospitalization and 2.8 times for hospital emergency treatment. In 2007 the rate of 15 to 29 year-old men was 92.8/100,000 inhabitants. The Southeast and Northeast have the highest incidence and prevalence. The conclusion was that the male/female differential rates occurs during adolescence, intensifies in early adulthood, and despite decreasing in intensity, continues until death. Cultural gender models and socio-structural aspects were examined to explain such marked differences.

Key words Aggression, Mortality, Morbidity, Emergency, Young
Resumo Analisa-se a mortalidade, internação hospitalar e atendimentos de emergência por agressão no Brasil, de 1996 a 2007. As fontes dos dados são o Sistema de Informação sobre Mortalidadel SIM, o Sistema de Informação Hospitalar/SIH e o Sistema de Vigilância das Violências e Acidentes/ VIVA, do Ministério da Saúde. Focaliza-se o sexo masculino e a faixa dos 15 aos 29 anos, além de outras variáveis referentes à vítima, ao agressor e ao evento. Encontram-se relações homem/mulher que são 11,6 vezes maiores na mortalidade, 4,5 vezes na internação e 2,8 vezes no atendimento de emergência. Em 2007, a taxa de mortalidade de homens de 15 a 29 anos foi 92,8/100.000 habitantes. As regiões Sudeste e Nordeste do país apresentam as maiores incidências e prevalências. Conclui-se destacando que o diferencial das taxas homem/mulher ocorre a partir da adolescência, se intensifica na idade adulta jovem e, embora perca intensidade, permanece até o final da vida. Recorre-se a modelos culturais de gênero, além de aspectos socioestruturais para explicar tão marcadas diferenças.

Palavras-chave Agressão, Mortalidade, Morbidade, Emergência, Jovem 


\section{Introdução}

A mortalidade por agressão configura-se como um grave problema de saúde pública no Brasil. Apesar da queda observada nas taxas de mortalidade por essa causa nos anos 2000, elas ainda são superiores às de alguns países, como a China ( 1,2 por $100.000 \mathrm{em} 2007)$ e a Argentina (5,2 por $100.000 \mathrm{em} \mathrm{2007)})^{1}$

Os homens, principalmente os mais jovens, ocupam papel central nas mortes por agressão, em todo o mundo. Em 2002, estimava-se que no mundo $80 \%$ das mortes por agressão haviam incidido sobre os homens. No Brasil, em 2005, esse percentual era de $92 \%{ }^{2}$. Em 2007, no país, 92\% dos homicídios eram masculinos, sendo que a principal faixa etária foi a de 20 a 29 anos, representando $40,3 \%$ do total desses óbitos ${ }^{1}$.

$\mathrm{O}$ fato de haver uma maior presença masculina nesses eventos específicos e na violência em geral, tem ocasionado um debate acerca do lugar e da condição de homens e mulheres nas sociedades $^{3}$. Discute-se que tanto os status quanto os papéis dos homens que os associam à violência se encontram relacionados a aspectos socialmente construídos. Desde cedo, meninos são levados a aprenderem e a reproduzirem comportamentos agressivos e violentos contra si mesmos e contra outrem $^{4}$. Alguns modelos culturais de masculinidade têm contribuído para que haja não só uma ligação entre gênero e violência, traduzida na hierarquia entre homens e mulheres, mas também eventos violentos ocorridos entre homens ${ }^{5}$. Para alguns autores, caminhando na contramão do processo civilizatório, haveria uma hipermasculinidade que poderia explicar as agressões cometidas por homens contra mulheres, assim como as mútuas entre homens ${ }^{6}$.

Neste estudo defende-se que o predomínio do sexo masculino no perfil epidemiológico de mortalidade por agressão pode ser explicado a partir da existência de modelos culturais de masculinidade. Com essa premissa, são analisados dados de mortalidade, internações hospitalares $e$ atendimentos de emergência em serviços sentinela no Brasil, e discutem-se, à luz da literatura, os fatores que fazem com que homens jovens sejam mais vulneráveis e tenham maior risco frente à violência.

\section{Metodologia}

Realizou-se uma análise epidemiológica de dados de mortalidade, internação hospitalar e de atendimento de emergência em serviços sentinela re- lativos às agressões (código X85 - Y09 da Classificação Internacional de Doenças - CID10) ocorridas no Brasil, no período de 1996 a 2007. As fontes pesquisadas foram o Sistema de Informação sobre Mortalidade/SIM, o Sistema de Informação Hospitalar/SIH e o componente inquérito do Sistema de Vigilância das Violências e Acidentes/VIVA, do Ministério da Saúde. Detalhou-se a análise no sexo masculino e na faixa dos 15 aos 29 anos, grupo mais afetado pelas agressões, considerando-se também outras variáveis referentes à vítima (raça/cor, escolaridade), ao agressor (sexo, relação com a vítima) e ao evento (tipo de agressão, meio usado, local, evolução do caso). A análise compreende o Brasil e suas grandes regiões. Apresentam-se frequências absolutas e relativas, e taxas de mortalidade e de internação hospitalar. As taxas foram calculadas tomando-se como base a população do IBGE. Os resultados são discutidos à luz das teorias de gênero.

\section{Resultados}

Panorama da morbimortalidade por agressão na população brasileira em geral

No período analisado percebe-se crescimento das taxas de mortalidade por agressão na população do país, do ano de 1996 (23,8/100.000 hab.) a $2003(28,2 / 100.000$ hab.), quando o índice foi o mais elevado. A partir de 2004 (26,4/ 100.000 hab.) a curva apresenta uma inflexão e as taxas decrescem desde então com pequenas oscilações, chegando em 2007 a 24,6/100.000 habitantes (Gráfico 1). A já elevada taxa masculina cresce $20,8 \%$, passando de 43,7 , em 1996, para 52,8/100.000 habitantes, em 2003. Em 2004 se inicia um processo descendente e assim permanece até o final do período. Em 2007, a taxa masculina era 46,2/100.000 habitantes tendo caído $12,5 \%$ em relação ao ano de 2003, e voltado ao patamar inicial da série estudada. No sexo feminino as taxas apresentam pequenas oscilações, de 4,4 em 1996, caindo, em 2007, para 3,9/100.000 habitantes (Gráfico 1).

Em média, o sexo masculino representa mais de $90 \%$ dos óbitos por essa causa. Suas taxas foram, em média, 11,5 vezes maiores que as femininas. Em 2002 e 2003 essa sobremortalidade masculina foi de 12,3 vezes, e em 1996 observouse seu menor valor (9,9 vezes).

No ano de 2007 foram registrados 46.652 óbitos por agressão no Brasil, numa relação de 11,6 homens para cada mulher morta por essa causa. O grupo mais atingido é o dos 20 aos 29 
anos, com a maior taxa de mortes por agressão, em ambos os sexos. Nessa faixa etária os homens têm risco 15,2 vezes maior que as mulheres. Os adolescentes dos 15 aos 19 anos respondem pela segunda maior taxa dessas mortes e os homens a apresentam 13,4 maior que a feminina (Tabela 1).

Considerando-se as regiões brasileiras para o período de 1996 a 2007, 52,9\% dos óbitos masculinos por agressão e 51,4\% dos femininos, ocor- reram no Sudeste; no Nordeste foram 23,9\% e $21,4 \%$, respectivamente; no Sul $(9,7 \%$ e $12,1 \%)$, no Centro-Oeste $(7,2 \%$ e $8,8 \%)$ e na região Norte $(6,3 \%$ em ambos os sexos).

Nesse mesmo ano, ocorreram 37.215 internações hospitalares por agressão no país, sendo $97,1 \%$ delas, de homens. A Tabela 1 mostra taxas de internação menores que as de mortalidade em ambos os sexos. Entretanto, embora com menor intensidade, prevalece a vitimização mascu-

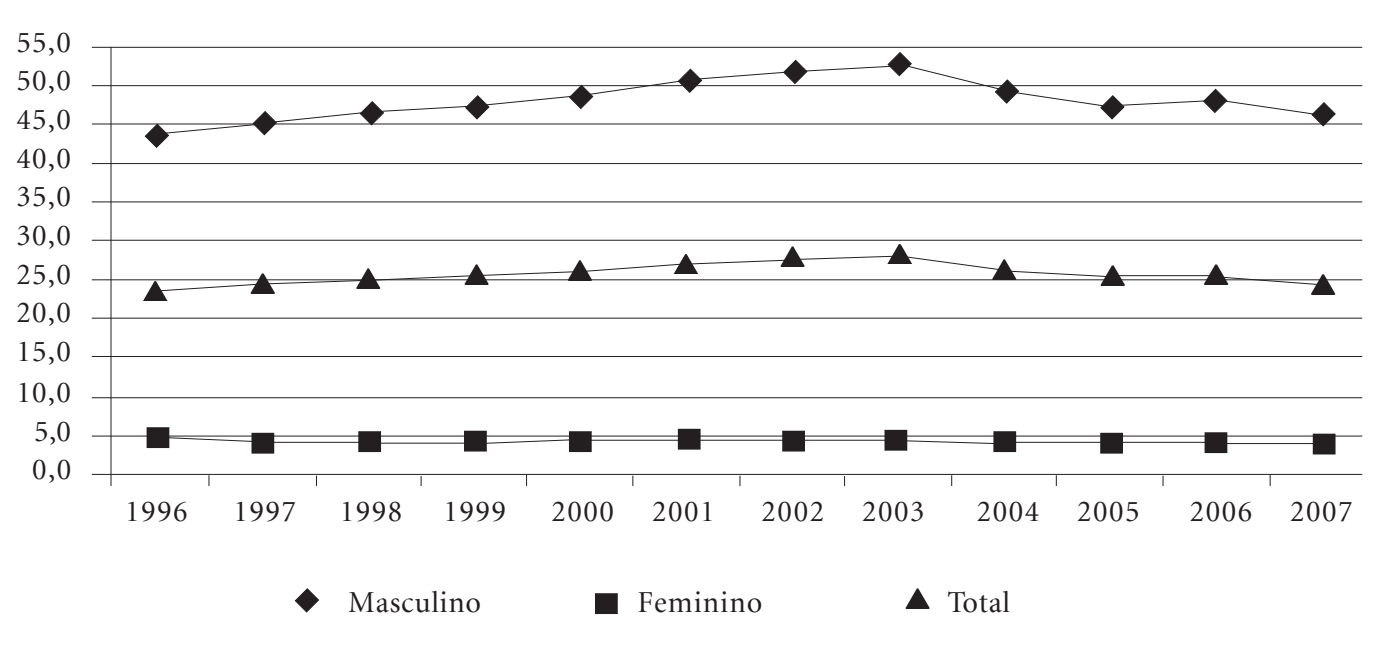

Gráfico 1. Taxas ${ }^{\star}$ de mortalidade por agressão (X85-Y09) segundo sexo. Brasil, 1996 a 2007.

(*) Taxas por 100.000 habitantes

Fonte: SIM/SVS/MS.

Tabela 1. Número e taxa de óbitos e internações, número e proporção dos atendimentos de emergência por agressão (X85 - Y09) segundo sexo e faixas etária. Brasil, 2007.

\begin{tabular}{|c|c|c|c|c|c|c|c|c|c|c|c|c|}
\hline \multirow{3}{*}{$\begin{array}{l}\text { Faixas } \\
\text { Etárias }\end{array}$} & \multicolumn{4}{|c|}{ Óbitos } & \multicolumn{4}{|c|}{ Internações } & \multicolumn{4}{|c|}{ Atendimentos de emergência } \\
\hline & \multicolumn{2}{|c|}{ Masculino } & \multicolumn{2}{|c|}{ Feminino } & \multicolumn{2}{|c|}{ Masculino } & \multicolumn{2}{|c|}{ Feminino } & \multicolumn{2}{|c|}{ Masculino } & \multicolumn{2}{|c|}{ Feminino } \\
\hline & No & Taxa $^{*}$ & No & $\operatorname{Taxa}^{*}$ & No & $\operatorname{Taxa}^{* *}$ & No & $\operatorname{Taxa}^{* *}$ & No & $\%^{* * *}$ & No & $\%^{* * *}$ \\
\hline $0-9$ & 178 & 1,0 & 121 & 0,7 & 1571 & 0,1 & 789 & 0,0 & 172 & 4,5 & 110 & 8,1 \\
\hline $10-14$ & 457 & 5,4 & 122 & 1,5 & 1301 & 0,2 & 430 & 0,1 & 161 & 4,2 & 95 & 7,0 \\
\hline $15-19$ & 6791 & 77,7 & 496 & 5,8 & 4569 & 0,5 & 731 & 0,1 & 569 & 15,0 & 190 & 14,0 \\
\hline $20-29$ & 17645 & 100,3 & 1167 & 6,6 & 12176 & 0,7 & 1778 & 0,1 & 1411 & 37,2 & 424 & 31,2 \\
\hline $30-39$ & 9340 & 67,8 & 810 & 5,6 & 7324 & 0,5 & 1361 & 0,1 & 784 & 20,6 & 294 & 21,6 \\
\hline $40-49$ & 4838 & 42,5 & 503 & 4,1 & 4760 & 0,4 & 1065 & 0,1 & 381 & 10,0 & 142 & 10,4 \\
\hline $50-59$ & 2263 & 28,8 & 250 & 2,9 & 2361 & 0,3 & 675 & 0,1 & 159 & 4,2 & 55 & 4,0 \\
\hline $60+$ & 1432 & 17,6 & 239 & 2,4 & 2065 & 0,3 & 1259 & 0,1 & 91 & 2,4 & 37 & 2,7 \\
\hline Total & 42944 & 46,2 & 3708 & 3,9 & 36127 & 0,4 & 1088 & 0,1 & $3728^{* * * *}$ & 100 & $1347^{* * * *}$ & 100 \\
\hline
\end{tabular}

* Taxa de óbitos por 100.000 mil habitantes; ${ }^{* *}$ Taxa de internação por 1.000 habitantes; ${ }^{* *}$ Proporção dos atendimentos por agressão em serviços sentinela de emergência; ${ }^{* * * *}$ Exclui 84 atendimentos de emergência com idade e sexo ignorados. 
lina, com uma relação de internação homem/ mulher de 4,5. Entre os homens, as maiores taxas de internação ocorreram também nos grupos etários dos 20 a 29 anos e dos 15 aos 19 anos, além da faixa dos 30 aos 39 anos. Já as taxas de internação das mulheres permaneceram praticamente constantes nos distintos grupos etários.

Do total das hospitalizações masculinas realizadas em 2007, as devidas à agressão representam 2,6\%. Entre as mulheres esse percentual é $0,1 \%$. No contexto das internações por causas externas, as proporções de internação por agressão são de $8,8 \%$ para os homens e $5,2 \%$ para as mulheres.

Em termos regionais, predominaram as internações no Sudeste (41,2\% masculinas e $44,7 \%$ femininas), seguidas pelo Nordeste $(34,6 \%$ e $30,9 \%)$, Sul (11,6\% e 12,2\%), Norte (7,3\% e 7,3\%) e Centro Oeste (5,4\% e 4,9\%). É importante ressaltar que parte dessas diferenças depende da capacidade instalada da rede de serviços que é distinta entre as regiões.

Ainda em 2007, dados do sistema VIVA inquérito apontam 5.159 registros de atendimento por agressão nos serviços sentinela de emergência, sendo $3.798(73,6 \%)$ masculinos, o que corresponde a uma relação de 2,8 homens para cada mulher. Nesse mesmo ano, 44,7\% desses atendimentos foram realizados na região Nordeste, seguida pelo Sudeste $(28,2 \%)$, Norte $(14,4 \%)$, Centro-Oeste $(9,0 \%)$ e o Sul $(3,8 \%)$.

Os indivíduos do sexo masculino com idade entre 20 a 29 anos respondem por $38 \%$ dos atendimentos de emergência. Entre as mulheres, predomina esse mesmo grupo etário $(31,0 \%)$, seguido pelos da faixa de 30 a 39 anos $(21,6 \%)$.

Tabela 2. Número e taxa de óbitos e internações, número e proporção dos atendimentos de emergência de homens de 15 a 29 anos por agressão (X85 - Y09) segundo as Regiões do Brasil, 2007.

\begin{tabular}{|c|c|c|c|c|c|c|}
\hline \multirow{2}{*}{ Região } & \multicolumn{2}{|c|}{ Óbitos } & \multicolumn{2}{|c|}{ Internações } & \multicolumn{2}{|c|}{$\begin{array}{l}\text { Atendimentos } \\
\text { de emergência }\end{array}$} \\
\hline & No & $\operatorname{Taxa}^{*}$ & No & $\operatorname{Taxa}^{* *}$ & No & $\%^{* * *}$ \\
\hline Norte & 2099 & 89,5 & 1391 & 0,6 & 343 & 17,3 \\
\hline Nordeste & 8404 & 108,3 & 6079 & 0,8 & 968 & 48,9 \\
\hline Centro-Oeste & 1836 & 96,1 & 977 & 0,5 & 177 & 9,0 \\
\hline Sudeste & 9115 & 85,6 & 6354 & 0,6 & 421 & 21,2 \\
\hline Sul & 2982 & 81,2 & 1944 & 0,5 & 71 & 3,6 \\
\hline Brasil & 24436 & 92,8 & 16745 & 0,6 & 1980 & 100,0 \\
\hline
\end{tabular}

* Taxa de óbitos por cem mil habitantes. ${ }^{* *}$ Taxa de internação por mil habitantes. ${ }^{* * *}$ Proporção dos atendimentos por agressão em serviços sentinela de emergência.

\section{Morbimortalidade de homens jovens (15 a 29 anos) por agressão}

Em 2007, a taxa de mortalidade de jovens por agressão foi de $92,8 / 100.000$ habitantes, totalizando 24.436 óbitos, o que representa $56,9 \%$ da mortalidade masculina por essa causa. As regiões Nordeste e Centro-Oeste apresentam o maior risco de morte de homens na faixa dos 15-29 anos por agressão (Tabela 2), enquanto a região Sul se destaca com o menor índice.

Os homens jovens totalizaram 16.745 internações por agressão em 2007, correspondendo a $46,3 \%$ das hospitalizações masculinas por essa causa (Tabela 2). Em relação a este evento, destacam-se a região Nordeste, com taxas superiores às nacionais, e a Sul e Centro-Oeste, com as taxas mais baixas do país.

Observa-se que no Brasil, em 2007, foram realizados 1.980 atendimentos de emergência a jovens do sexo masculino por agressão, representando $53,1 \%$ dos 3.798 atendimentos masculinos por esse evento. A região Nordeste apresentou a maior proporção desses atendimentos e o Sul a menor (Tabela 2).

As características sociodemográficas destes homens dos 15 aos 29 anos atendidos nos serviços de emergência por agressão, no ano de 2007, mostram que: $76,2 \%$ eram pardos ou pretos e $21,0 \%$ eram brancos; $32,3 \%$ possuíam de 4 a 7 anos de estudo (fundamental incompleto). A violência física predominou em $96,5 \%$ dos casos de agressão atendidos nesses serviços, seguida pela violência psicológica $(9,0 \%)$. O meio predominantemente usado na agressão foi a força física/ espancamento (49,7\%), que também despontou como líder em todas as regiões do país. Os objetos cortantes ou penetrantes $(32,6 \%)$ e as armas de fogo $(19,3 \%)$ foram meios também mais utilizados na agressão.

Cerca de $88 \%$ dos casos de agressão aos jovens atendidos nas emergências ocorreram na zona urbana, a maioria deles na via pública (53,3\%), mas também em bar ou similar (18,5\%) e na residência da vítima (13,8\%). Em 46,7\% desses eventos a vítima havia feito uso de álcool. Grande parcela dos jovens agredidos e atendidos nas emergências $(63,6 \%)$ evoluiu para alta, $22,7 \%$ tiveram encaminhamento hospitalar e $2,1 \%$ foram a óbito.

No que se refere ao agressor, $37,7 \%$ são conhecidos, 34,0\% são desconhecidos e 18,6\% são familiares das vítimas. Para $75,9 \%$ das mulheres, os principais são os familiares e conhecidos. Esses agressores representam para os homens $49,4 \%$. Por conseguinte, $16,2 \%$ dos agressores das 


\section{Discussão}

Os diferenciais observados na ocorrência de agressão, com desvantagem para o sexo masculino pode ser explicado a partir da influência de modelos culturais de gênero. A socialização de homens, desde cedo, costuma ancorar-se em um "conjunto de atributos, valores, funções e condutas que se espera que um homem tenha em uma determinada

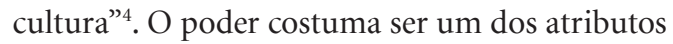
valorizados nessa socialização em determinadas sociedades e isso pode vir associado à violência, uma vez que esta pode ser vista como um instrumento para a obtenção do poder ou para a resistência a ele entre os segmentos masculinos ${ }^{5}$.

Outros traços valorizados nessa socialização também podem concorrer para que o sexo masculino esteja significativamente mais presente nos atos violentos do que o feminino. Zaluar ${ }^{6}$ chama atenção para a existência da 'hipermasculinidade', traduzida por um conjunto de traços - como a agressividade, a dominação, valorização do perigo, dentre outros - que pode levar homens a agredirem mulheres ou a se agredirem entre si tendo como referência esse ideal de virilidade.

Além do predomínio do sexo masculino nos casos de violência em geral, os dados deste estudo revelam uma gradação da vitimização masculina, comparada à feminina. Ela é mais intensa na mortalidade, menor na internação (casos severos de agressão) e ainda menor na atenção de emergência (casos menos graves), dando uma noção da intensidade da violência que incide sobre os homens.

No panorama brasileiro, em termos regionais, o Sudeste e o Nordeste se revezam como as regiões com maior representatividade no tocante aos três fenômenos investigados - morte, internação e atendimento de emergência por agressão na população em geral. As regiões Norte e Centro-Oeste aparecem como de menor incidência dessas mortes e de prevalência dessas internações hospitalares. Finalmente, a região Sul situa-se em níveis intermediários em relação às mortes e internações, mas é a que apresenta menores índices de atendimentos de emergência por agressão. $\mathrm{O}$ Nordeste também se destaca nos casos de agres-

são letal e não letal de homens jovens. Não por acaso, é no Sudeste onde se encontram as maiores cidades do país e do mundo, constituindo áreas urbanas com elevada densidade populacional, nas quais se estabelecem complexas e anômicas relações interpessoais. É sobretudo nos espaços públicos das vias e bares destas cidades, majoritariamente frequentados por homens, que não raro consomem bebidas alcoólicas, onde ocorre grande parcela das agressões letais e não letais. A estes fatores acresce a cultura machista, ainda amplamente predominante na região Nordeste, onde o bar e o signo do 'quem bebe mais é mais macho' permanecem estruturantes da cultura de gênero e da virilidade masculina.

A distinção entre os sexos também se faz notar no conjunto dos dados relativos às faixas etárias. O diferencial das taxas homem/mulher ocorre a partir da adolescência (15-19 anos), se intensifica na faixa seguinte (20-29) e, embora perca a intensidade, permanece até o final da vida. O envolvimento de jovens nos eventos violentos tem sido analisado à luz de aspectos socioestruturais. Nesse sentido, observa-se que:

más condições de vida, a falta de oportunidades para os jovens quanto à escolarização com qualidade e consequente dificuldades de inserção no mercado de trabalho formal, que refletem as enormes desigualdades socioeconômicas e de acesso a serviços básicos, aliadas à expansão do tráfico e consumo de drogas ilícitas para além dos grandes centros urbanos, também têm sido atribuídas como condicionantes para o aumento dos indicadores de mortalidade por homicídios ${ }^{7}$.

Além disso, para $\mathrm{Kouri}^{8}$, a forte participação dos jovens nos atos violentos pode ser visto a partir do fato de ser esse segmento o mais influenciado pela cultura da violência e do medo do outro, que ergue barreiras invisíveis às pessoas e as faz temer tudo e a todos. Segundo o autor:

Entre os jovens este embaraço ganha contornos mais nítidos, associado que está a um distanciamento maior e cada vez mais alongado do poder de consumo que vai desde o tempo e a qualidade da educação formal, à questão da inserção no mercado de trabalho precoce e cada vez mais difícil, até a aquisição de objetos de moda ${ }^{8}$.

No perfil das vítimas e dos principais agressores também se destacam os homens negros e aqueles com baixa escolaridade. Frente a esse fato, além do entendimento de modelos culturais de gênero, é preciso compreender outros aspectos estruturantes como a raça/etnia e a classe social. Soares Filho ${ }^{9}$, num estudo de série temporal dos homicídios no Brasil, de 2000 a 2009, constatou que, em termos de vitimização por homicídios, 
houve um aumento de $28,6 \%$ entre negros e, ao contrário, entre brancos houve uma redução de $24,5 \%$. Segundo o autor:

Como as condições de desvantagem social experienciadas pela população negra podem relacionar-se ao racismo e à discriminação, essas também podem se somar em desvantagens ainda na mortalidade, no caso o homicídio9.

A intensidade das armas de fogo na mortalidade e da agressão física encontradas nos dados podem também ser vistas a partir da simbologia masculina. Moura ${ }^{10}$ observa que o uso de armas de fogo pode ser entendido como uma construção de gênero. Segundo a autora, essa utilização é uma expressão da socialização de um tipo de masculinidade violenta e militarizada, fazendo parte, muitas vezes, de rituais que promovem o fascínio e familiaridade com e por esse artefato, no momento da passagem de rapazes para a fase adulta.

A compreensão dos modelos de gênero, em especial os relacionados às masculinidades, pode ajudar no entendimento de serem os homens, ao mesmo tempo, as principais vítimas e os principais agentes dos eventos violentos. Como lembra Bourdieu ${ }^{11}$, os homens - sem se perceberem - podem ser também vitimas, caindo nas arma- dilhas da dominação masculina. Assim, para afirmar a sua virilidade, eles podem agredir mulheres e outros homens ou se expor mais à violência do que as mulheres.

\section{Colaboradores}

ER Souza, R Gomes e JG Silva trabalharam na concepção, elaboração e redação final do texto; BSC Correia realizou a organização e análise dos dados e MMA Silva realizou a revisão final do texto.

\section{Agradecimento}

À Secretaria de Vigilância em Saúde, Departamento de Análise de Situação em Saúde, Coordenação Geral de Doenças e Agravos não Transmissíveis, Área Técnica de Vigilância e Prevenção de Violências e Acidentes pela cessão do banco de dados do Sistema VIVA.

\section{Referências}

1. Reichennheim ME, Souza ER, Moraes CL, Mello Jorege MHP, Silva CMF, Minayo MCS. Violência e lesões no Brasil: efeitos, avanços alcançados e desafios futuros. The Lancet 2011; 6736(11):75-89.

2. Camargo ABM. Mortes por causas violentas no estado de São Paulo: a influência das agressões. São Paulo em Perspectiva 2007; 21(1):31-45.

3. Souza ER. Masculinidade e violência no Brasil: contribuições para a reflexão no campo da saúde. Cienc Saude Colet 2005; 10(1):59-70.

4. Gomes R. Sexualidade masculina, gênero e saúde. Rio de Janeiro: Editora Fiocruz; 2008.

5. Greig A. Political connections: men, gender and violence. Santo Domingo: INSTRAW; 2001. (Working Paper, n. 1). [acessado 2008 nov 23]. Disponível em: http://www.instraw.org/en/docs/mensroles/Greig.pdf

6. Zaluar A. Agressão física e gênero na cidade do Rio de Janeiro. RBCS 2009; 24(71):9-22.

7. Lozada EMK, Mathias TAF, Andrade SM, Aidar T. Tendência da mortalidade por homicídios no Estado do Paraná, segundo Regionais de Saúde, 1979 a 2005. Rev Bras de Epidemiol 2009; 12(2):258-269.
8. Koury MG P. Cultura da violência e o medo do outro: observações sobre medos, violência e juventude no Brasil atual. Rev Antropologia Experimental 2004; 4:1-10.

9. Soares Filho AM. Vitimização por homicídios segundo características de raça no Brasil. Rev. Saude Publica 2011; 45(4):745-755.

10. Moura T. Masculinidades e feminilidades entre as (micro)guerras e as (macro)pazes: um estudo de caso sobre o Rio de Janeiro.[artigo da internnet]. [acesso 2008 nov 23]. Disponível em: http://www. eurozine.com/articles/2007-06-28-moura-pt.html

11. Bourdieu P. A dominação masculina. Rio de Janeiro: Bertrand Brasil; 1999.

Artigo apresentado em 10/05/2012

Aprovado em 23/07/2012

Versão final apresentada em 13/09/2012 